\title{
Noninvasive Diagnosis of Hepatocellular Carcinoma in Cirrhotic Liver: Current Guidelines and Future Prospects for Radiological Imaging
}

\author{
Jens Ricke Max Seidensticker Konrad Mohnike \\ Klinik für Radiologie und Nuklearmedizin, \\ Universitätsklinikum Magdeburg AöR, Magdeburg, Germany
}

\section{Key Words}

Diagnostic guidelines $\cdot \mathrm{HCC} \cdot$ Imaging $\cdot$ Liver $\cdot$ Liver cancer

\begin{abstract}
Noninvasive imaging has become the standard for hepatocellular carcinoma (HCC) diagnosis in cirrhotic patients. Typical imaging features of HCC such as arterial wash-in and venous wash-out deliver very high specificity and acceptable sensitivity even in nodules from 1 to 2 $\mathrm{cm}$ in diameter. However, limitations apply specifically in hypovascular HCC, for which the addition of new techniques such as diffusion-weighted magnetic resonance imaging (DW-MRI) or hepatobiliary MRI is helpful. Whereas DW-MRI adds to both the sensitivity and specificity, hepatobiliary MRI additionally contributes valuable information in cirrhotic patients on the histopathology of small lesions, including early HCC and high-grade dysplastic nodules. Biopsy of small, atypical lesions is associated with a high rate of false-negative findings and should be used only after careful consideration in selected patients. Here, we review the current international guidelines on HCC diagnosis as well as the latest developments in imaging that may contribute to safe detection and accurate characterization of suspicious nodules in patients with liver cirrhosis.




\section{Introduction}

Noninvasive imaging has become the worldwide standard for hepatpcellular carcinoma (HCC) diagnosis in cirrhotic patients. Diagnostic criteria are similar in international guidelines, such as those endorsed by the American Association for the Study of Liver Diseases (AASLD) or the Asian Pacific Association for the Study of the Liver (APASL), with regard to "classic" hypervascular HCC. However, there is less consensus on the diagnostic criteria for small, atypical or hypovascular HCC, leading to different approaches being applied in different countries. Here, we will review the current international guidelines on HCC diagnosis, including the latest developments in imaging that may contribute to safe detection and accurate characterization of suspicious nodules in patients with liver cirrhosis.

\section{Histopathology of HCC and Its Implications for Diagnostic Imaging}

HCC is the most frequent cause of death in patients with liver cirrhosis [1, 2]. Carcinogenesis of HCC in cirrhotic livers mirrors a continuous development from dysplastic foci to clonal expansion [3]. Typically, transformation continues from very small heterogeneous lesions with foci of highly differentiated carcinoma cells to poorly differentiated larger tumor nodules [4-6].

The prognosis of HCC patients is dependant on lesion size even at the very early tumor stages $[1,2,7]$. With increasing tumor size, portal invasion becomes frequent and has a suspected negative effect on prognosis [8]. The Japanese literature on the histopathology of HCC has introduced the classification "early HCC" [9-11]. Information about early HCC was derived from surgical specimens, leaving uncertainty as to what the natural course of these lesions might be. Early HCC is generally hypovascular up to $2 \mathrm{~cm}$ in size, with no vessel infiltration and with invasiveness limited to the periportal fields. Tumor borders are ill-defined, dysplasia is frequent, and unpaired arteries are rarely found. Small foci of carcinoma cells suggest that early HCCs are precursors of HCC [12]. In contrast to early HCC with ill-defined borders and favorable prognosis, Japanese pathologists also identified "small HCC." Small HCC has a sharp border demarcation, and microscopic portal invasion is frequent despite the small lesion size. Presumably, the prognosis of patients with small HCC is unfavorable as a result of early portal invasion and microsatellite formation, even though clinical evidence regarding prognosis is not available $[8,11,12]$.

\section{Guidelines for HCC Diagnosis}

Screening of cirrhotic patients primarily is intended to detect and classify small tumor nodules. Screening for HCC is most important in high-risk groups such as patients with cirrhosis or nonalcoholic steatohepatitis and carriers of hepatitis virus B or C with or without cirrhosis [13-15]. Small HCC nodules usually produce a hypoechogenic pattern on ultrasonography. In rare cases nodules may appear hyperechogenic because of steatosis developing with hypoxia during early carcinogenesis and ongoing vascular transformation [16]. Typical HCC hypervascularity develops in lesions reaching 1-2 cm in diameter and is common in those larger than $2 \mathrm{~cm}$. Dynamic contrast-enhanced imaging by computed tomography (CT) or magnetic resonance imaging (MRI) demonstrates not only arterial hyperperfusion, but an enhanced venous wash-out phenomenon compared with adjacent normal liver tissue. A pattern of combined arterial wash-in and venous wash-out has a specificity of almost $100 \%$ 


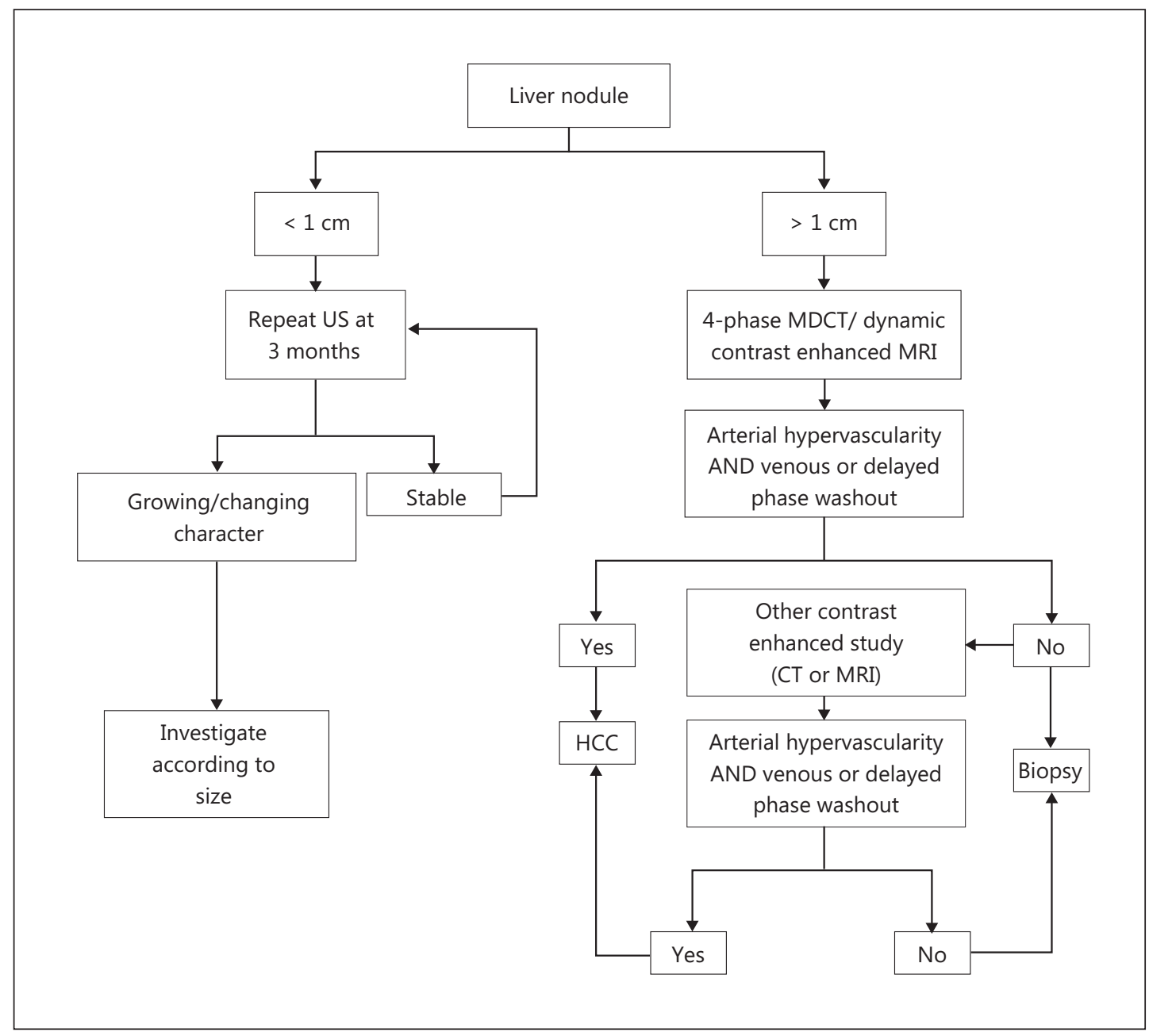

Fig. 1. Diagnostic algorithm of the AASLD (from [1]). MDCT = multidetector computed tomography; US = ultrasonography.

in tumors larger than $1 \mathrm{~cm}$. However, sensitivity in lesions measuring $1-2 \mathrm{~cm}$ is $44-62 \%$ with CT alone and 44-53\% with dynamic MRI alone [17-19].

Contrast-enhanced ultrasound (CEUS) also shows the typical wash-in and wash-out phenomena characterizing HCC [20]. However, in contrast to CT or MRI, CEUS gives this pattern for cholangiocellular carcinoma also. In such cases, additional imaging by MRI or CT is necessary to show that venous wash-out is present, thus excluding cholangiocellular carcinoma [21]. Consequently, a positive result during CEUS has no clinical significance (i.e., it will be followed by MRI or CT). As a result, CEUS has been removed from the guidelines of the AASLD $[1,2]$.

In addition to the guidelines of the AASLD, other international recommendations for the diagnosis of HCC exist. Typically, a hypoechogenic lesion is detected during ultrasound examination of a cirrhotic patient. International guidelines such as those from the AASLD or the APASL do not have major differences regarding the diagnosis of classical hyperperfused HCC (fig. 1,2). Both guidelines support noninvasive diagnosis based on a typical pattern of arterial wash-in and venous wash-out. In the AASLD guidelines, the threshold for lesion work up is 1 $\mathrm{cm}[1,2]$. Lesions smaller than $1 \mathrm{~cm}$ should undergo surveillance by ultrasound at 3-month intervals. Either MRI or CT findings demonstrating the typical vascular pattern of HCC are 


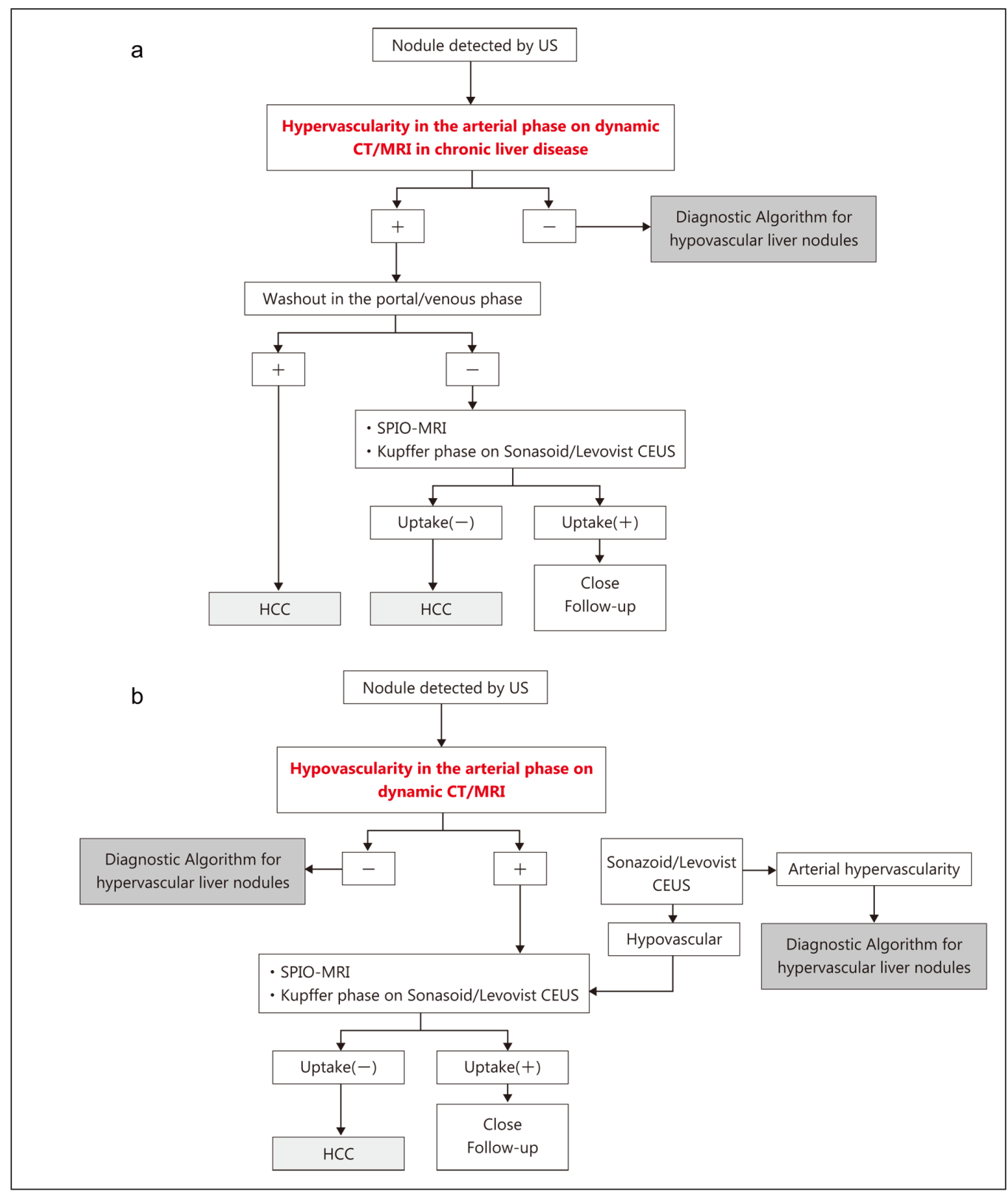

Fig. 2. Diagnostic algorithm of APASL for hypovascular lesions (from [23]).SPIO, superparamagnetic iron oxide; CEUS, contrast-enhanced ultrasonography.

sufficient for a definitive diagnosis. Lesion biopsy is recommended only for suspicious nodules without wash-in and wash-out and after confirmation by a second alternative imaging technique (i.e., MRI after an initial CT). This approach algorithm enables noninvasive HCC diagnosis in patients with liver cirrhosis for a lesion size larger than $1 \mathrm{~cm}$ with very high specificity. In a study of lesions from 1- to 2-cm in diameter by Forner et al. [17], MRI demonstrated a specificity of $96.6 \%$ and a sensitivity of $62 \%$ using the AASLD criteria. Leoni et al. studied a cohort of $43 \mathrm{HCC}$ lesions of the same size. Using the same criteria (wash-in and wash-out with at least one imaging method), specificity was $87 \%$ and sensitivity was $70 \%$. In tumors of 2-3 cm, sensitivity improved to $96 \%$ [22].

In contrast to the AASLD criteria, APASL criteria do not stratify with respect to nodule 
size [23]. APASL differentiates between hypo- and hypervascular lesions. Hypovascular nodules larger than $1 \mathrm{~cm}$ are especially demanding. In a study by Bolondi et al. of 72 lesions in cirrhotic livers, 41 had a maximum diameter of 1-2 cm and 31 lesions had a maximum diameter of $2.1-3 \mathrm{~cm}$ [24]. Of the tumor nodules $1-2 \mathrm{~cm}$ in diameter, $44 \%$ demonstrated arterial wash-in in two independent examinations; of the other $56 \%$ of lesions $1-2 \mathrm{~cm}$ in diameter, $50 \%$ demonstrated a hypovascular pattern in at least one of two imaging methods, and $50 \%$ demonstrated a hypovascular pattern in two imaging techniques. In essence, $20 \%$ of lesions displayed a hypovascular pattern in 2 imaging techniques, but only $64 \%$ of these were benign. This study was undertaken using the old EASL criteria, which do not consider the wash-out pattern. However, even if the AASLD criteria had been applied, hypovascular tumor characteristics would have led to a $35 \%$ false-negative rate in this selected cohort of tumors between 1 and $2 \mathrm{~cm}$ in diameter.

\section{The Role of Biopsy in HCC}

Tumor seeding is a major concern of clinicians when considering biopsy of suspected HCC. In a meta-analysis by Silva et al., tumor seeding in the puncture tract was found after $2.7 \%$ of biopsies [25]. In another meta-analysis in which Perkins et al. analyzed 99 articles, the risk for tumor seeding was calculated at $2.3 \%$. Interestingly, tumor seeding in the puncture tract seems to be reduced by around $50 \%$ with thermal tumor ablation such as radiofrequency ablation. During such interventions, the puncture tract usually is ablated during retraction of the radiofrequency probe, thus minimizing the risk of cell seeding [26, 27].

A practical issue of lesion biopsy in small tumors is the risk of false-negative findings. Forner et al. described 30\% false negatives at first biopsy of lesions smaller than $2 \mathrm{~cm} \mathrm{[17].}$ A second biopsy confirmed the false-negative finding in $61 \%$ of cases. Another limitation is that on histopathology, 35\% of high grade-dysplastic nodules (HGDNs) demonstrate islets of HCC cells, which are not necessarily included in the sample acquired by a small percutaneous biopsy needle [19, 24].

\section{The Emerging Role of Hepatobiliary MRI}

In recent years, hepatobiliary MRI employing gadolinium ethoxybenzyl diethylenetriamine pentaacetic acid (Gd-EOB-DTPA) (Primovist or Eovist) or gadobenate dimeglumine (Gd-BOPTA) (Multihance) has gained increasing attention. Gd-EOB-DTPA is of specific interest because of its favorable preference for biliary excretion compared with Gd-BOPTA. Whereas $50 \%$ of Gd-EOB-DTPA on average is eliminated via the biliary path (the other $50 \%$ undergoes renal elimination), GD-BOPTA undergoes only around $8 \%$ biliary excretion. The higher excretion rate with Gd-EOB-DTPA leads to a significantly higher signal intensity in hepatocytes, allowing distinct forecasts of the histopathological properties of a given lesion [28, 29]. Gd-EOB-DTPA is taken up by functional hepatocytes employing the organic anion transporting polypeptide (OATP) mechanism [30]. The latency period until uptake is sufficient for hepatobiliary imaging is $20 \mathrm{~min}$, with dynamic imaging similar to standard contrast MR being performed during that interval [31, 32]. HCC, precursor lesions of HCC, or benign lesions originating from hepatic tissue such as focal nodular hyperplasia or adenoma demonstrate a gradual uptake of Gd-EOB-DTPA, depending on their degree of differentiation (in the case of HCC) or, more specifically, the functionality of their OATP transporter mechanism. The uptake leads to a signal increase in T1-weighted sequences. 
A close correlation between functional OATP and histopathological grading from lowgrade dysplastic nodules (LGDN) via HGDNs to differentiated HCC has been shown in a number of studies. Well differentiated HCCs commonly demonstrate functional OATP with significant Gd-EOB-DTPA uptake in tumor cells [33, 34]. Since biliary ducts are missing in such lesions, Gd-EOB-DTPA will be retained and the signal enhancement is very intense [30, 33-38]. In a study by Golfieri et al. of 127 cirrhotic patients, 62 lesions were examined using dynamic and hepatobiliary MRI. Of these lesions, 20 were HGDNs or early HCC, 21 were LGDNs, 17 were regenerative nodules, and 4 were nodular regenerative hyperplasia. All findings were confirmed by histopathology. The inclusion of Gd-EOB-DTPA hepatobiliary late-phase imaging to dynamic contrast-enhanced sequences improved the sensitivity, specificity, accuracy, and positive and negative predictive values to greater than $95 \%$ (i.e., 88.4-99.4\%, 88-95\%, 88-98.5\%, 97-99\%, 65-97.5\%, respectively). Of these 62 lesions, 20 displayed signs of malignancy on histopathology (32\% were early HCC or HGDN), with 19 diagnosed by hepatobiliary MRI alone. Golfieri et al. concluded that hepatobiliary MRI increased the sensitivity of the diagnosis of HGDN or early HCC by $11 \%$. The negative predictive value was increased by $32.5 \%$ [39]. Based on these results, the current guidelines of the Japan Society of Hepatology (JSH) have already incorporated the use of Gd-EOB-DTPA and Sonazoid ultrasound for atypical hypovascular lesions [4].

In a study that used hepatobiliary MRI in patients with Child-Pugh A cirrhosis who had undergone liver transplantation or resection, Kim et al. found a significant correlation of the signal intensity with poorly, moderately, and highly differentiated HCC [35]. In a longitudinal study, Akai et al. followed hypovascular lesions rated nonmalignant despite no uptake of GdEOB-DTPA in the hepatobiliary phase: 97 lesions measured 5-10 mm, 24 lesions measured 11-15 $\mathrm{mm}$ and 9 lesions were larger than $15 \mathrm{~mm}$. Over time, confirmed transformation to HCC occurred in $3.2 \%, 11.1 \%$, and $15.9 \%$ at 1,2 , and 3 years [40]. In a similar patient cohort, Kumada et al. described a change of perfusion patterns of hypovascular lesions to arterial hyperperfusion - a surrogate for malignant transformation - in $27.6 \%$ and $43.5 \%$ of lesions at 6 and 12 months [41].

\section{Diffusion-Weighted MRI}

Diffusion-weighted magnetic resonance imaging (DW-MRI) of the liver has also gained increasing attention in recent years. In DW-MRI, intracellular proton movement is visualized and displayed as a parametric image. A number of studies have evaluated both the sensitivity and specificity of DW-MRI with respect to suspected HCC in cirrhosis [42]. In addition, the method has the potential to assess tumor response after the treatment of solid tumors with systemic or local therapies that induce structural changes in tumor cells [43]. However, DW-MRI is highly sensitive to field inhomogeneities and tissue movements, making liver imaging especially challenging. Recently, Vandecaveye et al. compared dynamic MRI employing wash-in and wash-out criteria with DW-MRI (with b values of b0, b100, b600, and b1000). Hepatobiliary MRI was not performed. For detection of malignant lesions, DWMRI with b600-SI(ratio) yielded a sensitivity of 95.2\% (compared to $80.6 \%$ for conventional MRI) and a specificity of $82.7 \%$ (compared to $65.4 \%$ ). In lesions smaller than $2 \mathrm{~cm}$, DW-MRI demonstrated improved sensitivity compared with conventional MRI ( $91.2 \%$ versus $67.6 \%$ ), improved specificity ( $82.9 \%$ versus $61 \%$ ), improved positive predictive value $(81.6 \%$ versus $59 \%$ ), and improved negative predictive value (91.9\% versus $69.4 \%$ ) [44]. 


\section{Conclusions}

Noninvasive imaging with either CT or MRI yields very high specificity with an acceptable sensitivity even for HCCs smaller than $2 \mathrm{~cm}$ if arterial wash-in and arterial wash-out criteria are applied. However, atypical hypovascular HCCs may be frequently missed; for such atypical lesions, the addition of new techniques such as DW-MRI or hepatobiliary imaging is advantageous. Whereas DW-MRI enhances both the sensitivity and specificity, hepatobiliary MRI frequently yields valuable additional information on the histopathology of small lesions, including early HCC and HGDN, in cirrhotic patients. However, the inclusion of hepatobiliary MRI in international guidelines (other than the Japanese guidelines) is still pending. Biopsy of small, atypical lesions is associated with a high rate of false-negative findings and should be used after careful consideration in selected patients only.

\section{References}

1 Bruix J, Sherman M: Management of hepatocellular carcinoma: an update. Hepatology 2011;53:1020-1022.

2 Bruix J, Sherman M: Management of hepatocellular carcinoma. Hepatology 2005;42:1208-1236.

3 Efremidis SC, Hytiroglou P, Matsui O: Enhancement patterns and signal-intensity characteristics of small hepatocellular carcinoma in cirrhosis: pathologic basis and diagnostic challenges. Eur Radiol 2007;17:2969-2982.

4 Kudo M, Izumi N, Kokudo N, et al: Management of hepatocellular carcinoma in Japan: Consensus-Based Clinical Practice Guidelines proposed by the Japan Society of Hepatology (JSH) 2010 updated version. Dig Dis 2011;29:339-364.

5 KojiroM: Pathological diagnosis at early stage: reaching international consensus. Oncology 2010;78(Suppl 1):31-35.

6 Roskams T, Kojiro M: Pathology of early hepatocellular carcinoma: conventional and molecular diagnosis. Semin Liver Dis 2010;30:17-25.

7 Lu XY, Xi T, Lau WY, et al: Pathobiological features of small hepatocellular carcinoma: correlation between tumor size and biological behavior. J Cancer Res Clin Oncol 2011;137:567-575.

8 Shi M, Zhang CQ, Zhang YQ, Liang XM, Li JQ: Micrometastases of solitary hepatocellular carcinoma and appropriate resection margin. World J Surg 2004;28:376-381.

9 Sakamoto M, Hirohashi S, Shimosato Y: Early stages of multistep hepatocarcinogenesis: adenomatous hyperplasia and early hepatocellular carcinoma. Hum Pathol 1991;22:172-178.

10 SakamotoM: Pathology of early hepatocellular carcinoma. Hepatol Res 2007;37(Suppl 2):S135-S138.

11 Takayama T, Makuuchi M, Kojiro M, et al: Early hepatocellular carcinoma: pathology, imaging, and therapy. Ann Surg Oncol 2008;15:972-978.

12 Nakashima Y, Nakashima O, Tanaka M, Okuda K, Nakashima M, Kojiro M: Portal vein invasion and intrahepatic micrometastasis in small hepatocellular carcinoma by gross type. Hepatol Res 2003;26:142-147.

13 Trevisani F, D'Intino PE, Morselli-Labate AM, et al: Serum alpha-fetoprotein for diagnosis of hepatocellular carcinoma in patients with chronic liver disease: influence of HBsAg and anti-HCV status. J Hepatol 2001;34:570-575.

14 Trevisani F, Santi V, Gramenzi A, et al: Surveillance for early diagnosis of hepatocellular carcinoma: is it effective in intermediate/advanced cirrhosis? Am J Gastroenterol 2007;102:2448-2457.

15 Evans AA, Chen G, Ross EA, Shen FM, Lin WY, London WT: Eight-year follow-up of the 90,000-person Haimen City cohort: I. Hepatocellular carcinoma mortality, risk factors, and gender differences. Cancer Epidemiol Biomarkers Prev 2002;11:369-376.

16 Rimola J, Forner A, Tremosini S, et al: Non-invasive diagnosis of hepatocellular carcinoma $2 \mathrm{~cm}$ in cirrhosis. Diagnostic accuracy assessing fat, capsule and signal intensity at dynamic MRI. J Hepatol 2012.

17 Forner A, Vilana R, Ayuso C, et al: Diagnosis of hepatic nodules $20 \mathrm{~mm}$ or smaller in cirrhosis: Prospective validation of the noninvasive diagnostic criteria for hepatocellular carcinoma. Hepatology 2008;47:97104.

18 Sangiovanni A, Manini MA, Iavarone M, et al: The diagnostic and economic impact of contrast imaging techniques in the diagnosis of small hepatocellular carcinoma in cirrhosis. Gut 2010;59:638-644.

19 Khalili K, Kim TK, Jang HJ, Yazdi LK, Guindi M, Sherman M: Indeterminate 1-2-cm nodules found on hepatocellular carcinoma surveillance: biopsy for all, some, or none? Hepatology 2011;54:2048-2054.

20 Vilana R, Forner A, Bianchi L, et al: Intrahepatic peripheral cholangiocarcinoma in cirrhosis patients may display a vascular pattern similar to hepatocellular carcinoma on contrast-enhanced ultrasound. Hepatology 2010;51:2020-2029.

21 Rimola J, Forner A, Reig M, et al: Cholangiocarcinoma in cirrhosis: absence of contrast washout in delayed phases by magnetic resonance imaging avoids misdiagnosis of hepatocellular carcinoma. Hepatology 2009;50:791-798. 
22 Leoni S, Piscaglia F, Golfieri R, et al: The impact of vascular and nonvascular findings on the noninvasive diagnosis of small hepatocellular carcinoma based on the EASL and AASLD criteria. Am J Gastroenterol 2010;105:599-609.

23 Omata M, Lesmana LA, Tateishi R, et al: Asian Pacific Association for the Study of the Liver consensus recommendations on hepatocellular carcinoma. Hepatol Int 2010;4:439-474.

24 Bolondi L, Gaiani S, Celli N, et al: Characterization of small nodules in cirrhosis by assessment of vascularity: the problem of hypovascular hepatocellular carcinoma. Hepatology 2005;42:27-34.

25 Silva MA, Hegab B, Hyde C, Guo B, Buckels JA, Mirza DF: Needle track seeding following biopsy of liver lesions in the diagnosis of hepatocellular cancer: a systematic review and meta-analysis. Gut 2008;57:15921596.

26 PerkinsJD: Seeding risk following percutaneous approach to hepatocellular carcinoma. Liver Transpl 2007;13:1603-1607.

27 Stigliano R, Marelli L, Yu D, Davies N, Patch D, Burroughs AK: Seeding following percutaneous diagnostic and therapeutic approaches for hepatocellular carcinoma. What is the risk and the outcome? Seeding risk for percutaneous approach of HCC. Cancer Treat Rev 2007;33:437-447.

28 Lee JM, Zech CJ, Bolondi L, et al: Consensus report of the 4th International Forum for Gadolinium-Ethoxybenzyl-Diethylenetriamine Pentaacetic Acid Magnetic Resonance Imaging. Korean J Radiol 2011;12:403415.

29 Filippone A, Blakeborough A, Breuer J, et al: Enhancement of liver parenchyma after injection of hepatocyte-specific MRI contrast media: a comparison of gadoxetic acid and gadobenate dimeglumine. J Magn Reson Imaging 2010;31:356-364.

30 Narita M, Hatano E, Arizono S, et al: Expression of OATP1B3 determines uptake of Gd-EOB-DTPA in hepatocellular carcinoma. J Gastroenterol 2009;44:793-798.

31 Zech CJ, Grazioli L, Breuer J, Reiser MF, Schoenberg SO: Diagnostic performance and description of morphological features of focal nodular hyperplasia in Gd-EOB-DTPA-enhanced liver magnetic resonance imaging: results of a multicenter trial. Invest Radiol 2008;43:504-511.

32 Huppertz A, Haraida S, Kraus A, et al: Enhancement of focal liver lesions at gadoxetic acid-enhanced MR imaging: correlation with histopathologic findings and spiral CT-initial observations. Radiology 2005;234:468-478.

33 Kitao A, Matsui O, Yoneda N, et al: The uptake transporter OATP8 expression decreases during multistep hepatocarcinogenesis: correlation with gadoxetic acid enhanced MR imaging. Eur Radiol 2011;21:20562066.

34 Kitao A, Zen Y, Matsui O, et al: Hepatocellular carcinoma: signal intensity at gadoxetic acid-enhanced MR Imaging-correlation with molecular transporters and histopathologic features. Radiology 2010;256:817826.

35 Kim HY, Choi JY, Kim CW, et al: Gd-EOB-DTPA-enhanced MRI predicts the histological grade of hepatocellular carcinoma only in patients with child-pugh a cirrhosis. Liver Transpl 2012.

36 Suh YJ, Kim MJ, Choi JY, Park YN, Park MS, Kim KW: Differentiation of hepatic hyperintense lesions seen on gadoxetic acid-enhanced hepatobiliary phase MRI. AJR Am J Roentgenol 2011;197:W44-W52.

37 Chou CT, Chen YL, Wu HK, Chen RC: Characterization of hyperintense nodules on precontrast T1-weighted MRI: utility of gadoxetic acid-enhanced hepatocyte-phase imaging. J Magn Reson Imaging 2011;33:625632.

38 KudoM: Multistep human hepatocarcinogenesis: correlation of imaging with pathology. J Gastroenterol 2009;44(Suppl 19):112-118.

39 Golfieri R, Renzulli M, Lucidi V, Corcioni B, Trevisani F, Bolondi L: Contribution of the hepatobiliary phase of Gd-EOB-DTPA-enhanced MRI to Dynamic MRI in the detection of hypovascular small $(</=2 \mathrm{~cm}) \mathrm{HCC}$ in cirrhosis. Eur Radiol 2011;21:1233-1242.

40 Akai H, Matsuda I, Kiryu S, et al: Fate of hypointense lesions on Gd-EOB-DTPA-enhanced magnetic resonance imaging. Eur J Radiol 2012.

41 Kumada T, Toyoda H, Tada T, et al: Evolution of hypointense hepatocellular nodules observed only in the hepatobiliary phase of gadoxetate disodium-enhanced MRI. AJR Am J Roentgenol 2011;197:58-63.

42 Löwenthal D, Zeile M, Lim WY, et al: Detection and characterisation of focal liver lesions in colorectal carcinoma patients: comparison of diffusion-weighted and Gd-EOB-DTPA enhanced MR imaging. Eur Radiol 2011;21:832-840.

43 Dudeck O, Zeile M, Wybranski C, et al: Early prediction of anticancer effects with diffusion-weighted MR imaging in patients with colorectal liver metastases following selective internal radiotherapy. Eur Radiol 2010;20:2699-2706.

44 Vandecaveye V, De Keyzer F, Verslype C, et al: Diffusion-weighted MRI provides additional value to conventional dynamic contrast-enhanced MRI for detection of hepatocellular carcinoma. Eur Radiol 2009;19:2456-2466. 